\title{
Strain-threshold- and frequency-dependent seismic simulation of nonlinear soils
}

\author{
Duguo Wang $\cdot$ Chenggang Zhao
}

Received: 28 July 2014/ Accepted: 11 October 2014/Published online: 3 December 2014

(C) The Seismological Society of China, Institute of Geophysics, China Earthquake Administration and Springer-Verlag Berlin Heidelberg 2014

\begin{abstract}
A one-dimensional equivalent linear method (EQL) is widely used in estimating seismic ground response. For this method, the shear modulus and damping ratio of inelastic soil are supposed to be frequency independent. However, historical earthquake records and laboratory test results indicate that nonlinear soil behavior is frequencydependent. Several frequency-dependent equivalent linear methods (FDEQL) related to the Fourier amplitude of shear strain time history have been developed to take into account the frequency-dependent soil behavior. Furthermore, the shear strain threshold plays an important role in soil behavior. For shear strains below the elastic shear strain threshold, soil behaves essentially as a linear elastic material. To consider the effect of elastic-shear-strain-thresholdand frequency-dependent soil behavior on wave propagation, the shear-strain-threshold- and frequency-dependent equivalent linear method (TFDEQL) is proposed. A series of analyses is implemented for EQL, FDEQL, and TFDEQL methods. Results show that elastic-shear-strain-thresholdand frequency-dependent soil behavior plays a great influence on the computed site response, especially for the highfrequency band. Also, the effect of elastic-strain-thresholdand frequency-dependent soil behavior on the site response is analyzed from relatively weak to strong input motion, and results show that the effect is more pronounced as input motion goes from weak to strong.
\end{abstract}

D. Wang $(\bowtie) \cdot$ C. Zhao

School of Civil Engineering, Beijing Jiaotong University,

No.3 Shangyuancun Rd., Beijing 100044, China

e-mail: wangduguo@163.com

D. Wang

China Earthquake Disaster Prevention Center, No.9 Minzuyuan Rd., Beijing 100029, China
Keywords Nonlinear soil behavior - Strain threshold · Frequency-dependent $\cdot$ Site response

\section{Introduction}

It is well known that local site conditions exert great influence on propagated ground motions. One-dimensional site response analysis is widely used to account for local site effects. In one-dimensional seismic ground response analyses, soil deposits are assumed to be horizontally layered over a uniform half-space. The incident waves are assumed to be vertically propagating shear waves. The EQL method represented by SHAKE (Schnabel et al. 1972) is one of the most frequently used methods in the engineering practice. SHAKE91 (Idriss and Joseph 1992) is the modified version of this program. The advantages of the EQL model include small computational effort and few input parameters. For the EQL method, approximate linear solutions are obtained by assuming depth-dependent values for shear modulus and damping, which remain constant for the duration of the earthquake simulation. These properties are chosen at the beginning of each iteration so as to be consistent with the levels of strain computed in the previous iteration. Although the iterative linear algorithm often provides good results for engineering purposes, it has a number of disadvantages, including underestimation of amplification ratio in high-frequency region and divergence in deconvolution analysis under strong ground motion (Sugito 1995; Miura et al. 2000; Assimaki and Kausel 2002; Kausel and Assimaki 2002; Yoshida et al. 2002; Kwak et al. 2008; Hashash et al. 2010). To overcome these disadvantages, FDEQL methods were proposed, in which soil properties in individual layers are adjusted on a frequency-to-frequency basis to account for the strong 
variation of shear strain amplitude with frequency (Sugito 1995; Assimaki and Kausel 2002; Kausel and Assimaki 2002; Yoshida et al. 2002; Jiang and Xing 2007). The effective strain is expressed as a function of frequency, and the difference between various FDEQL methods is the relationship between effective strain and frequency.

Strain amplitude is a key variable in predicting soil behavior. Based on conceptual frameworks of soil behavior and published experiment data related to stress and strain thresholds, researchers have proposed alternative strain regime divisions and strain thresholds that represent the change in soil behavior (Anderson and Richart 1976; Vucetic and Dobry 1991; Vucetic 1994; Lanzo et al. 1997; Hsu and Vucetic 2003; Rahhal and Lefebvre 2006; Okur and Ansal 2007; Díaz-Rodríguez and López-Molina 2008; Guo 2012), one of which is defined as elastic threshold shear strain $\left(\gamma_{\text {te }}\right)$ (Kim et al. 1991; Okur and Ansal 2007). For shear strains below $\gamma_{\text {te }}$, soil behaves essentially as an elastic material. Therefore, for components of ground motion with strain amplitudes below $\gamma_{\text {te }}$, the shear modulus does not degrade, and the damping is very small. Based on the FDEQL method and elastic shear strain threshold concept, elastic-shear-strain-threshold- and frequencydependent (TFDEQL) soil behavior is proposed in this paper. A series of analyses is performed to investigate the effect of TFDEQL soil behavior on site response.

\section{Frequency-dependent modulus and damping}

For the EQL method, some percentage of peak shear strain is used to determine the shear modulus and damping ratio. Seed and Idriss (Schnabel et al. 1972) suggested that the effective shear strain $\left(\gamma_{\text {eff }}\right)$ is fixed as $65 \%$ of the maximum strain $\left(\gamma_{\max }\right)$, namely, $\gamma_{\text {eff }}=0.65 \gamma_{\max }$. Currently, an expression proposed by Idriss and Sun (Idriss and Joseph 1992) that relates the ratio of the effective shear strain to maximum shear strain $\left(R_{\gamma}\right)$ with the earthquake magnitude $(M)$ is commonly used.

$R_{\gamma}=\frac{M-1}{10}$.

However, the EQL method has been known to be unreliable in the case of propagating strong ground motion or soft soil deposits. Generally, the ground motions include some spectral characteristics, and the contribution of the frequency contents to strain time history strongly depends on the value of the frequency. Since the strong spectral characteristics of shear strain amplitude are included in the seismic ground response, it may be derived that an appropriate shear modulus and damping ratio, which depend on the frequency characteristic could be used for EQL analysis. Based on this assumption, various FDEQL algorithms were proposed to improve the accuracy of the approximate solution and better simulate the wide range of shear strains within the duration of the seismic loading (Sugito 1995; Assimaki and Kausel 2002; Kausel and Assimaki 2002; Yoshida et al. 2002; Jiang and Xing 2007).

Suigto (1995) proposed that the frequency-dependent equivalent strain can be derived using the following equation:

$\gamma_{\mathrm{f}}(\omega)=C \gamma_{\max } \frac{F_{\gamma}(\omega)}{F_{\gamma_{\max }}}$,

where $C=$ constant, $\gamma_{\max }=$ maximum shear strain, $F_{\gamma}(\omega)=$ Fourier spectrum of shear strain, and $F_{\gamma \max }$ represents the maximum of $F_{\gamma}(\omega)$. The definition of $\gamma_{\mathrm{f}}(\omega)$ is the equivalent strain, which controls the equivalent shear modulus and damping ratio and is proportional to the spectral amplitude of shear strain in the frequency domain. The constant $C$ controls the level of equivalent strain uniformly along the frequency axis.

Yoshida et al. (2002) pointed out that an apparent frequency dependence for the stiffness and damping ratio appears in the dynamic response. Following this observation, the effective strain is expressed in terms of frequency from the similarity concept of the strain-frequency relationship between the time domain and frequency domain. Equation (3) was proposed to evaluate an effective strain, from which the shear modulus and damping ratio are computed among various functions.

$\left\{\begin{array}{ll}\gamma_{\mathrm{eff}}=\gamma_{\max } & f_{\mathrm{p}}>f \\ \gamma_{\mathrm{eff}}=\gamma_{\max }\left[1-\left(\frac{\lg f-\lg f_{\mathrm{p}}}{\lg f_{\mathrm{e}}-\lg f_{\mathrm{p}}}\right)^{m}\right] & f_{\mathrm{p}} \leq f \leq f_{\mathrm{e}} \\ \gamma_{\mathrm{eff}}=0 & f>f_{\mathrm{e}}\end{array}\right.$,

where $f_{\mathrm{p}}$ is inverse of the period $(T)$ when shear strain is at maximum by the zero crossing method, $f_{\mathrm{e}}$ is the frequency above which nonlinear behavior need not be considered, and $m$ is a parameter. Effective strain is expressed by $m$ th order polynomial equation of $\lg f\left(\lg\right.$ is described as $\left.\log _{10}\right)$ between $f_{\mathrm{p}}$ and $f_{\mathrm{e}}$ and is constant outside this frequency range.

Kausel and Assimaki (2002) indicated that the EQL analyses disregard the fact that small-amplitude, high-frequency components of deformation involve hysteresis loops with little modulus degradation or damping. Thus, motions computed at the ground surface with the EQL method usually exhibit unrealistically low amplitudes at high frequencies. A smoothed strain spectrum, expressed by Eq. (4), was proposed to evaluate the effective strain to obtain the shear modulus and damping ratio.

$$
\begin{aligned}
\gamma_{\text {eff }} & =\gamma_{\max }\left|\frac{\gamma(\omega)}{\gamma_{0}}\right| \\
& =\left\{\begin{array}{ll}
\gamma_{\max } \\
\gamma_{\max }\left(\exp \left(-\alpha \frac{\omega}{\omega_{0}}\right) /\left(\frac{\omega}{\omega_{0}}\right)^{\beta}\right) & \omega>\omega_{0}
\end{array},\right.
\end{aligned}
$$



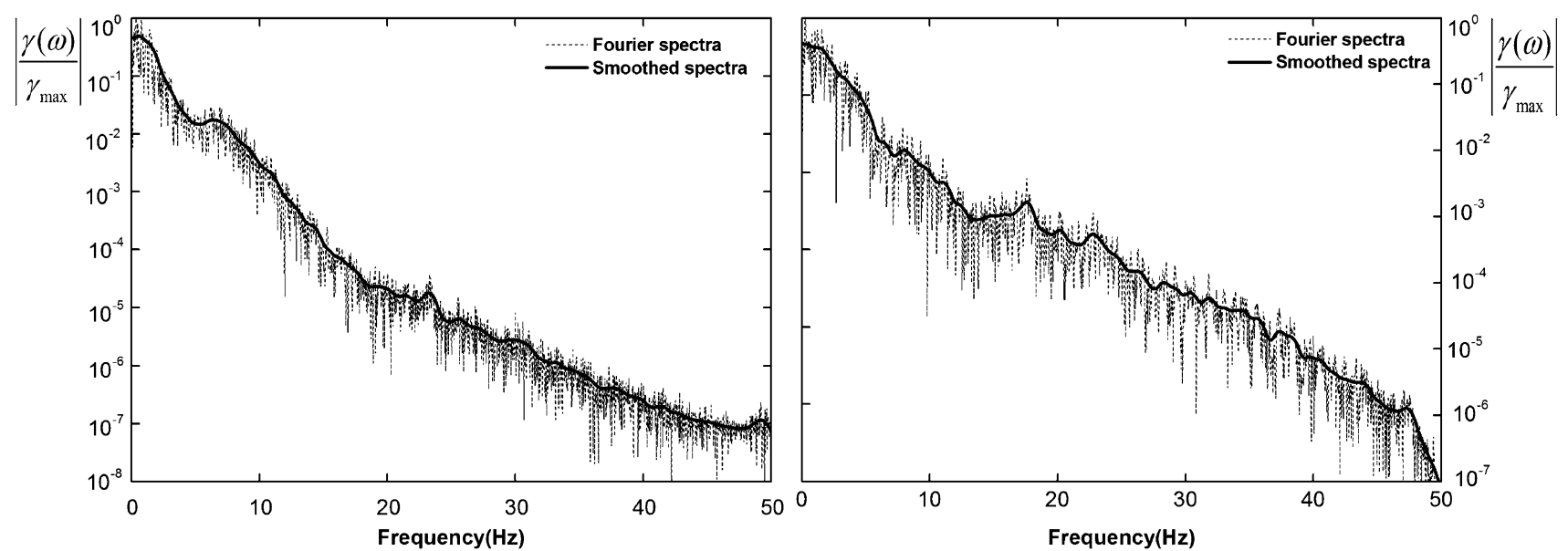

Fig. 1 Composite of strain Fourier spectra and smoothed spectra for the Kobe (left) and Northridge (right) earthquakes

where $\quad \omega_{0}=\int_{0}^{\infty} \omega \gamma(\omega) \mathrm{d} \omega / \int_{0}^{\infty} \gamma(\omega) \mathrm{d} \omega, \quad \gamma_{0}=1 / \omega_{0} \int_{0}^{\omega_{0}}$ $\gamma(\omega) \mathrm{d} \omega$, and the two parameters $\alpha$ and $\beta$ can be calculated through a simple least-squares optimal fit.

Jiang and Xing (2007) proposed a moving average method to fit the line of the strain Fourier amplitude spectrum and performed a series of analyses to evaluate the effect of frequency-dependent soil behavior on site response. Results show that the prediction obtained by the FDEQL method is better than that obtained by the EQL method.

The moving average method is evaluated next in this paper. In order to keep the energy of ground motion unchangeable when the moving average method is used, the Parzen's spectral window (Ohsaki 1994) is applied to smooth the strain spectrum. Thus, the effective shear strain can be derived by Eq. (5).

$\gamma_{\mathrm{eff}}(\omega)=\gamma_{\max } \frac{F_{\gamma}(\omega)}{F_{\gamma_{\max }}}$

where $\gamma_{\max }=$ maximum shear strain, $F_{\gamma}(\omega)=$ smoothed Fourier spectrum of shear strain, and $F_{\gamma \max }$ represents the maximum of $F_{\gamma}(\omega)$. The definition of $\gamma_{\text {eff }}(\omega)$ is the effective strain, which controls the equivalent shear modulus and damping factor and is proportional to the spectral amplitude of shear strain in frequency domain.

Figure 1 shows a composite of the normalized Fourier amplitude spectra of the strain time histories for the Kobe and Northridge earthquakes. The smoothed strain spectrum is also shown in Fig. 1.

\section{Elastic shear strain threshold}

Shear strain amplitude plays a key role in the determination of soil behavior in geotechnical earthquake engineering. The shear modulus and damping ratio vary significantly with the amplitude of shear strain under cyclic loading. To investigate the small-strain damping and elastic strain threshold, Kim et al. (1991) performed torsional shear (TS) and resonant column (RC) tests on 10 soils including both sand and cohesive soils with different plasticity index (PI) and confining pressures. Results show that an "elastic" zone was found. Through accurately measuring small-strain hysteresis loops in the TS test, the elastic threshold strains were found to range between 0.0001 and $0.02 \%$. Okur and Ansal (2007) conducted a total of 98 stress-controlled cyclic triaxial tests on normally consolidated and slightly over consolidated samples to investigate the effects of the PI and confining pressures on the dynamic behavior of soil. The soil samples used were taken from various sites in Turkey after the 1999 Kocaeli Earthquake as a part of post-earthquake investigations. According to test results, correlations were established for the elastic shear strain threshold in terms of the PI.

$\gamma_{\mathrm{te}}=\frac{0.00035}{1+11.92 \exp (-0.1 S)}$

where $\gamma_{\text {te }}=$ elastic shear strain threshold and $S=$ PI. Also, the relationship between dynamic shear modulus and damping ratio was established.

$D=-0.18114\left(\frac{G}{G_{\max }}\right)+0.20033$

where $D=$ damping ratio, $G_{\max }=$ small strain shear modulus, and $G=$ shear modulus compatible with shear strain. This definition of elastic threshold is adopted in this paper, and Eq. (6) is used to consider the relationship between elastic threshold and PI due to the availability of data (Okur and Ansal 2007). 


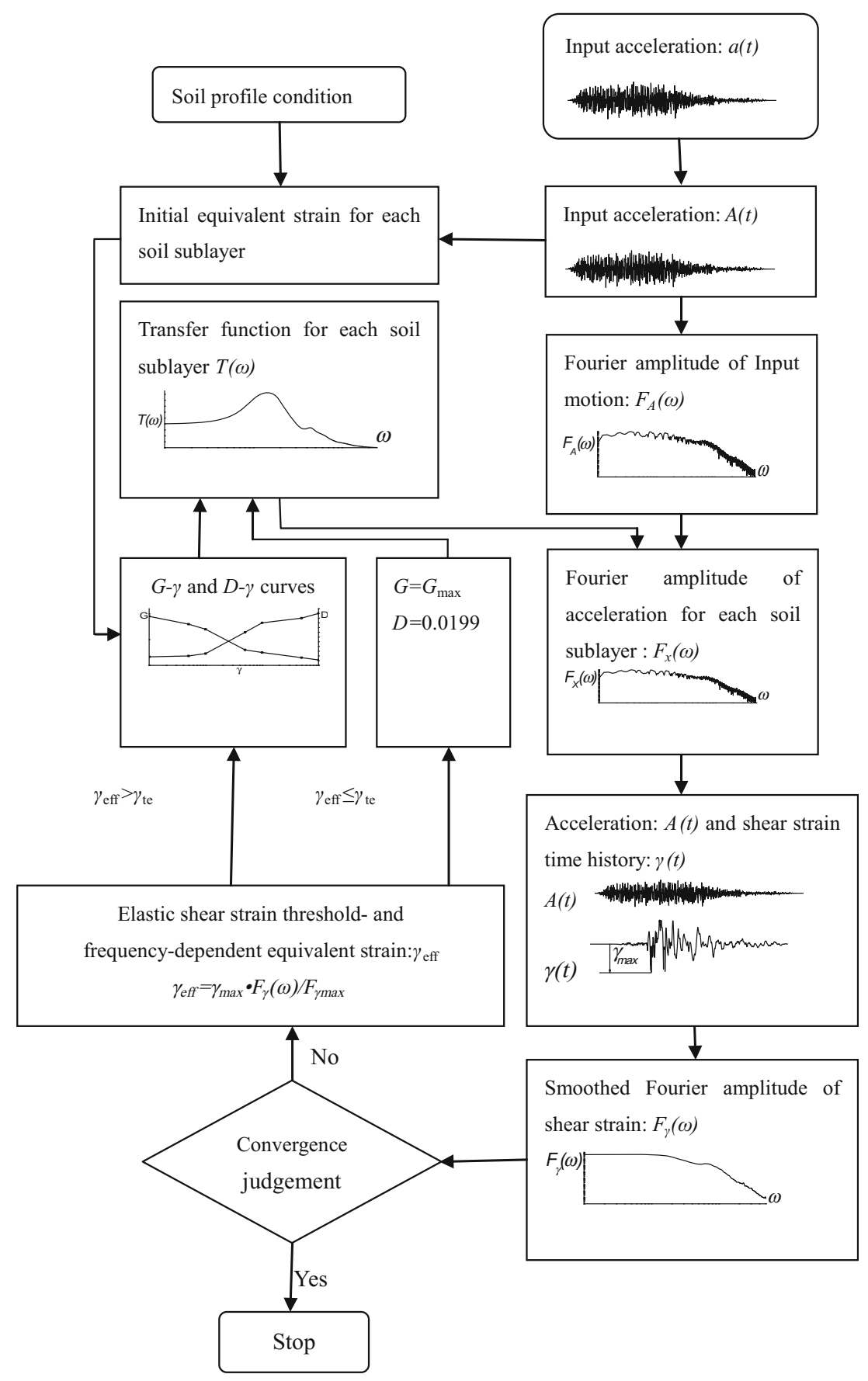

Fig. 2 Flow chart of iterative procedure for TFDEQL algorithm

\section{Elastic-threshold-dependent and frequency- dependent soil model}

Kausel and Assimaki (2002) indicated that the low-amplitude, high-frequency components of strain induce secondary loops, which are much narrower and more elastic in nature than the fundamental loop, i.e., they have a greater secant modulus and much less damping. Therefore, the shear strain modulus and damping ratio are supposed to be different for various frequency components. For soft soil deposits supported by rock or much stiffer soil, the seismic response of the soft soil at small strains can be very sensitive to variations in the small-strain shear modulus and damping because the stress-strain behavior of the soil is practically linear and the site is susceptible to large dynamic amplification in this strain range (Vucetic and Dobry 1991). If the soil is subjected to shear strain below the elastic shear strain threshold, Kim et al. 1991) pointed out that the dynamic shear modulus remains practically constant and is assumed to be equal to its initial value $G_{\max }$, which is adopted in 

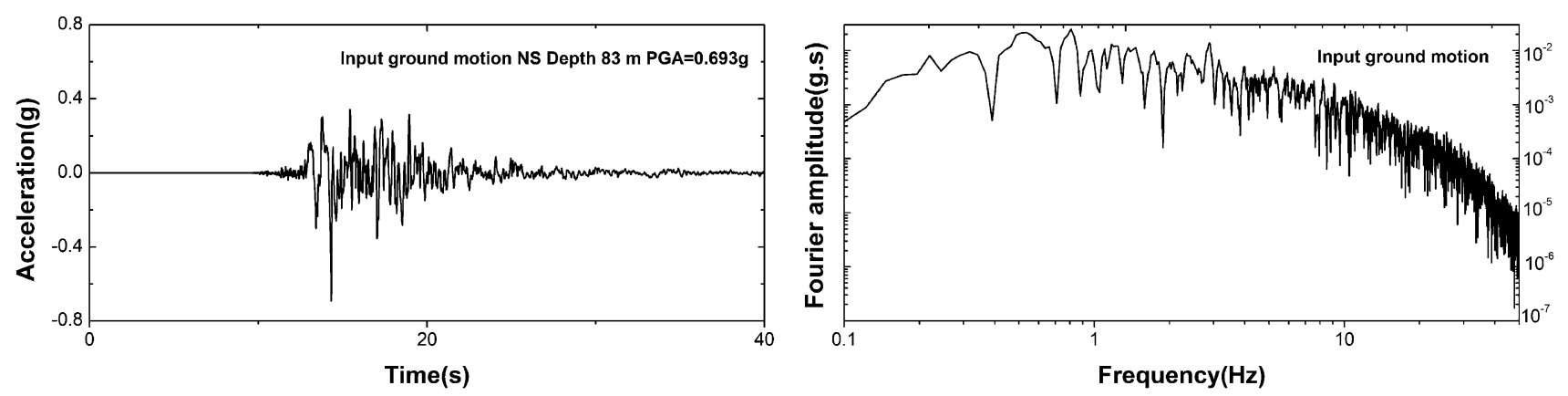

Fig. 3 Record at $83 \mathrm{~m}$ depth used as input motion: time history (left) and Fourier spectra (right)
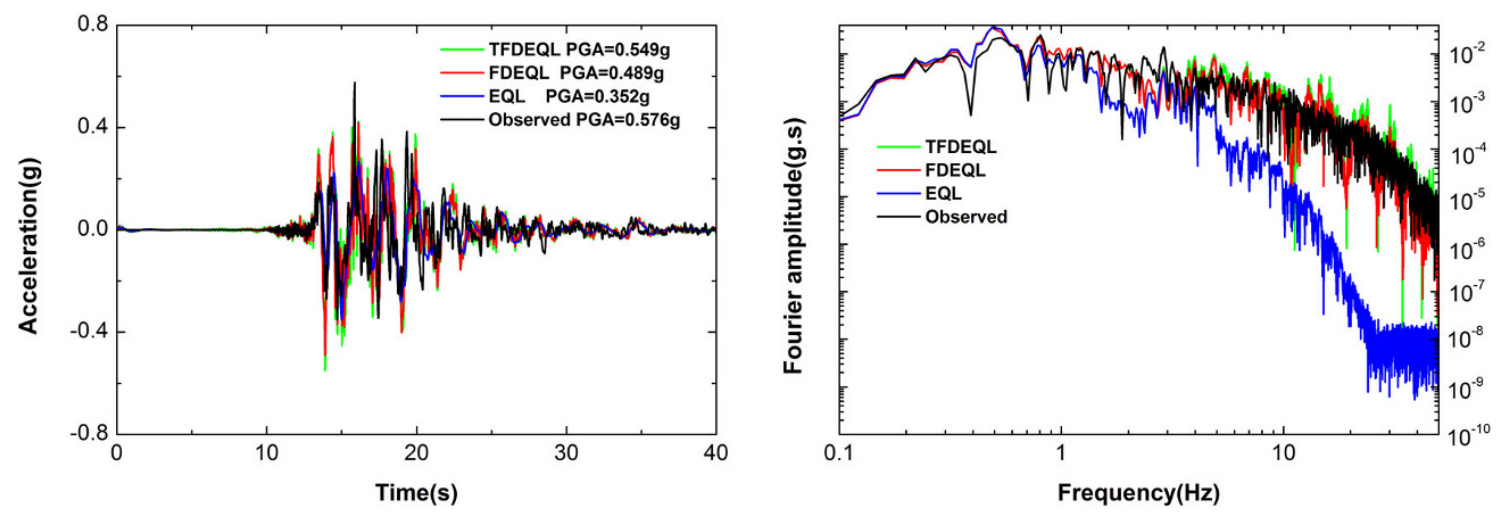

Fig. 4 Computed and observed time histories (left) and Fourier spectra (right) of the response at $16 \mathrm{~m}$ depth

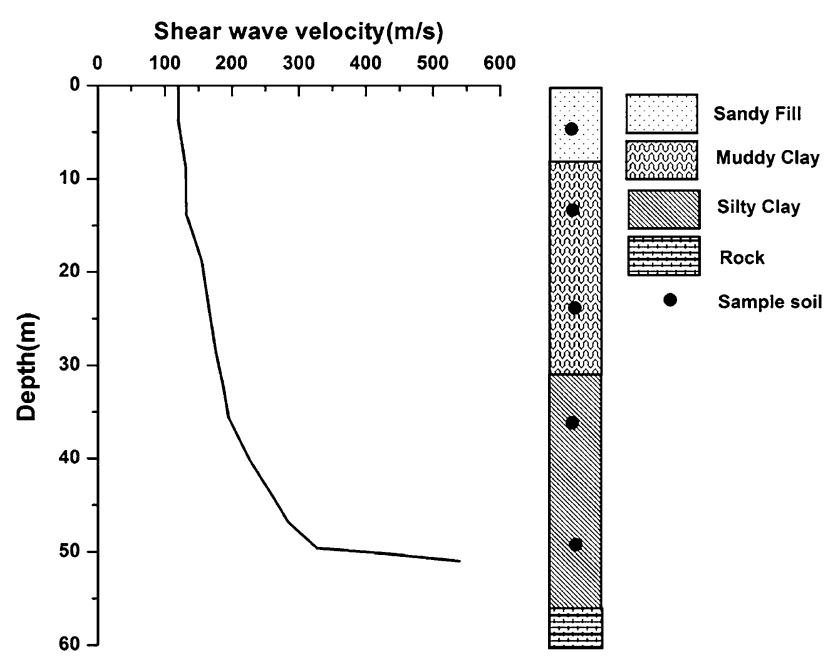

Fig. 5 Soil profile used in this paper

determining the dynamic shear modulus at strains below the elastic shear strain threshold. Theoretically, no dissipation of energy takes place at strains below the elastic threshold strain amplitude. However, a number of experimental studies indicated that some energy is dissipated even at very low strain levels (Lanzo and Vucetic 1999). Thus, the damping ratio is never zero. In this paper, the damping ratio $(D)$ is calculated by Eq. (7) with the condition that $G$ is equal to $G_{\max }$, and thus $D=0.01919$ if the shear strain is below elastic shear strain.

Based on the elastic shear strain threshold theory and frequency-dependent soil characteristics, the TFDEQL method is proposed. For the smoothed shear strain Fourier spectra, if the shear strain amplitude is below the elastic shear strain, the shear modulus is supposed to be $G_{\max }$, and the damping ratio is 0.01919; if the shear strain amplitude is beyond the elastic shear strain, the effective strain is derived by Eq. (5), and the shear modulus and damping can be calculated according to shear modulus and damping reduction curve.

\section{Elastic-threshold- and frequency-dependent soil behavior algorithm for site response}

The equivalent linear procedure (Schnabel et al. 1972) is an iterative procedure to obtain values for the modulus and damping ratio that are compatible with equivalent uniform strain induced in each sublayer. At the outset, a set of properties (shear modulus, damping, and total unit weight) is assigned to each sublayer of the soil deposit. The analysis is conducted using these properties, and the shear strains induced in each sublayer are calculated. The shear modulus and the damping ratio for each sublayer are then 

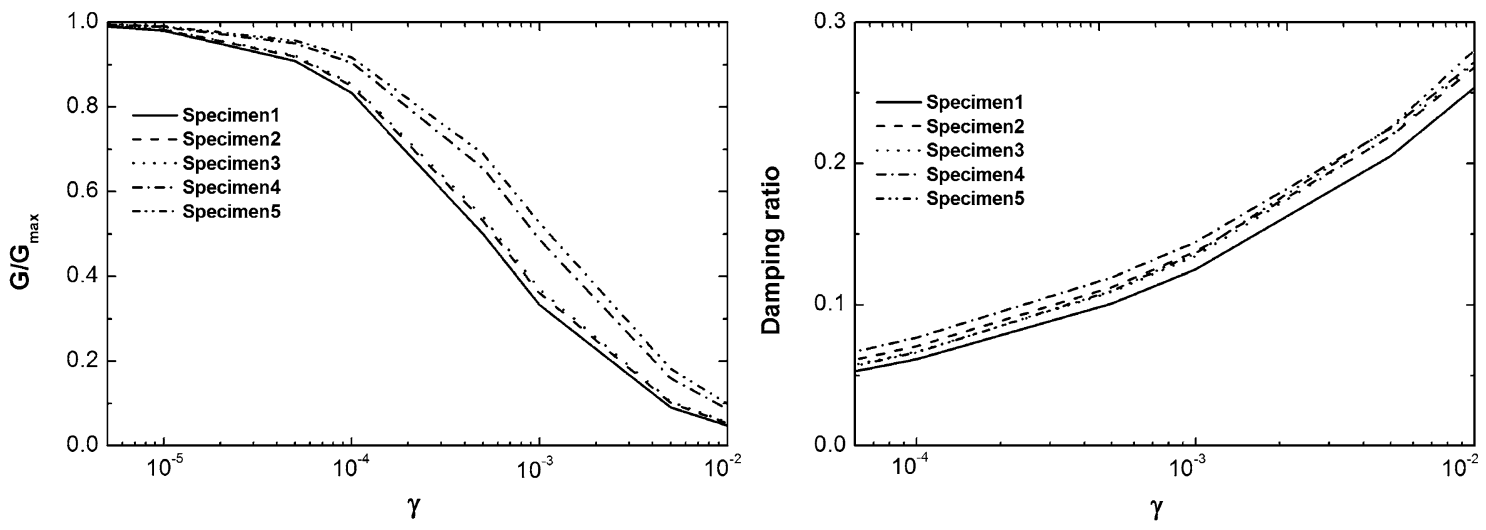

Fig. $6 G / G_{\max }-\gamma$ and $D-\gamma$ curves for five representative soil specimens
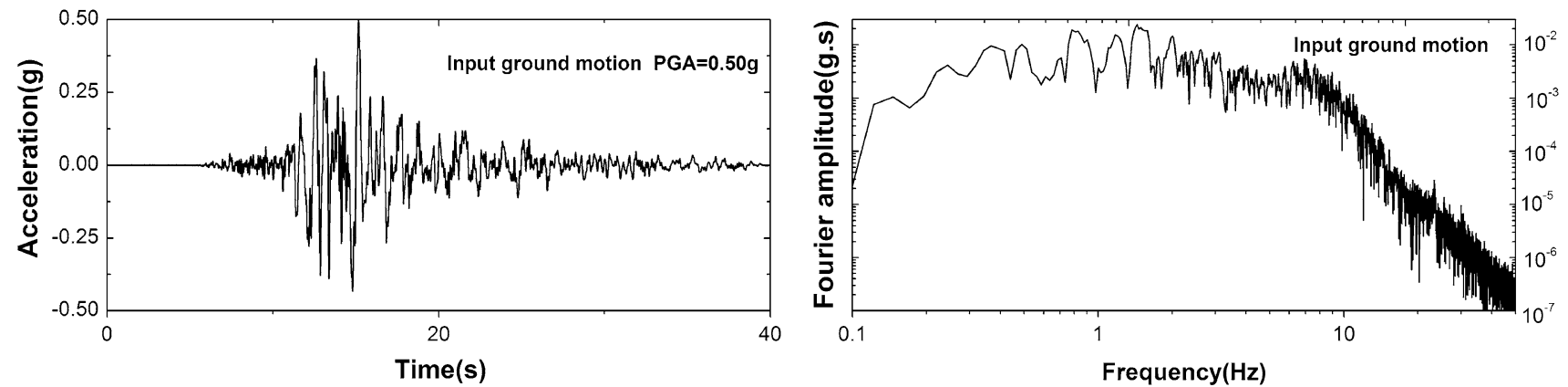

Fig. 7 Input motion used in site response analysis: time history (left) and Fourier spectra (right)
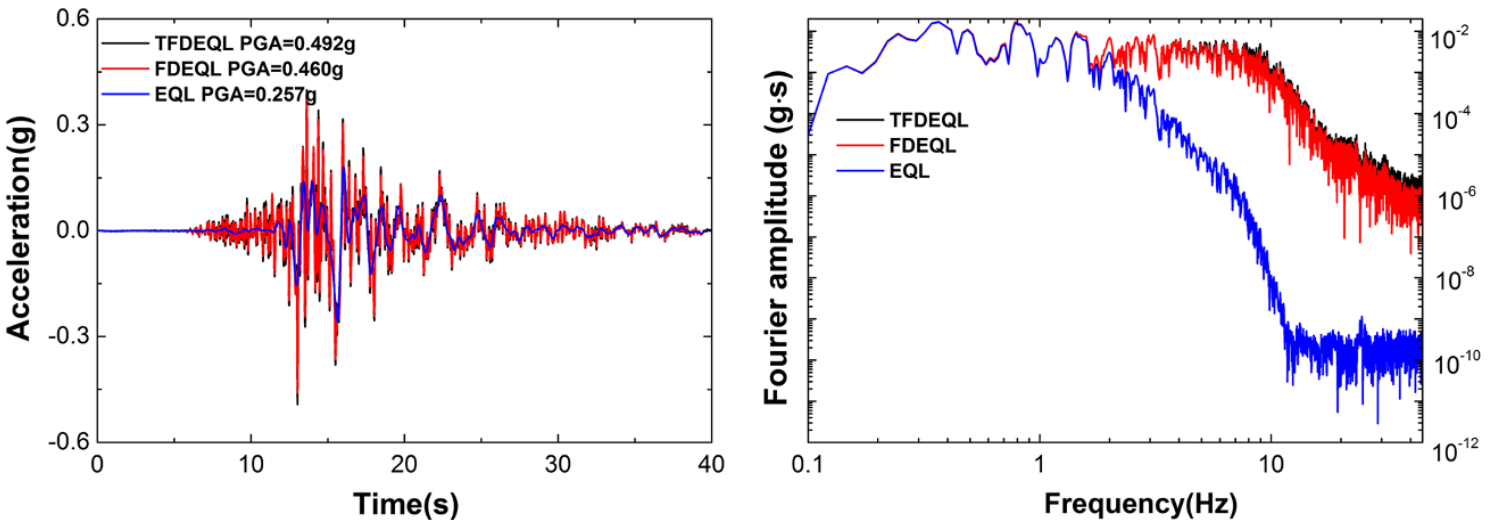

Fig. 8 Computed time histories (left) and Fourier spectra (right) of response at free surface

modified based on the relationship relating these two properties to shear strain. The analysis is repeated until strain-compatible modulus and damping values are achieved. Several FDEQL algorithms were proposed to account for the frequency-dependent behavior of soils (Sugito 1995; Assimaki and Kausel 2002; Kausel and Assimaki 2002; Yoshida et al. 2002; Jiang and Xing 2007). The TFDEQL algorithm is almost identical to the FDEQL algorithm, except that the shear modulus is $G_{\max }$ and the damping ratio is 0.01919 for shear strains below the elastic shear strain threshold. Based on the FDEQL algorithms, the flow chart of the iterative procedure for the TFDEQL algorithm is shown in Fig. 2.

In order to demonstrate the effectiveness of the TFDEQL method, simulations for three methods are conducted on vertical array observations during strong ground shaking. In this study, vertical array, strong-motion records, obtained at the reclaimed Port Island, Kobe during the 1995 Kobe earthquake, are utilized to evaluate the efficiency of the TFDEQL method. The array consisted of three-component accelerometers located at the surface and at depths of 16, 32, and $83 \mathrm{~m}$ You et al. (2009). indicated the soil profile and the 

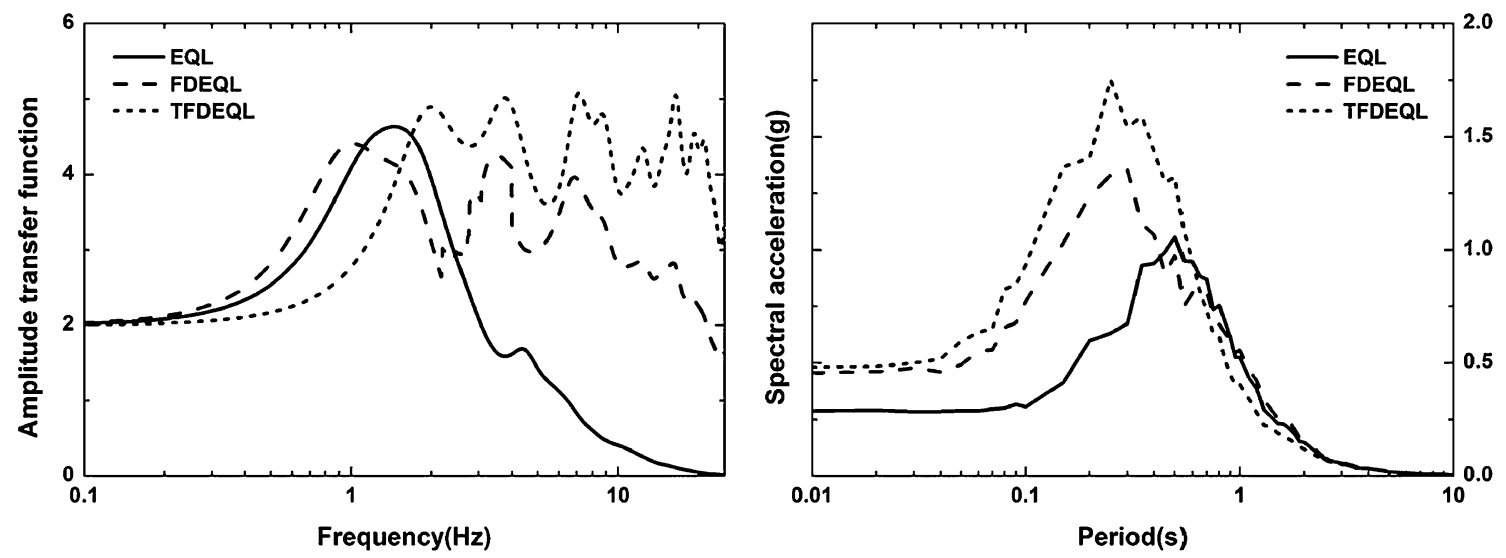

Fig. 9 Amplitude transfer function from rock to surface (left) and $5 \%$ damped surface acceleration response spectra (right) for the profile

Table 1 Maximum value of response spectra and corresponding periods for three models

\begin{tabular}{lll}
\hline Model & Maximum response spectra $(\mathrm{g})$ & Period $(\mathrm{s})$ \\
\hline EQL & 1.057 & 0.50 \\
FDEQL & 1.353 & 0.25 \\
TFDEQL & 1.753 & 0.25 \\
\hline
\end{tabular}

shear modulus reduction and damping ratio curves. Due to the liquefaction of the surface soil, the records obtained at the surface soil are not appropriate for calibrating the simulations. As a result, the records at depths of $16 \mathrm{~m}$ and $83 \mathrm{~m}$ are used. The record at $83 \mathrm{~m}$, shown in Fig. 3, is regarded as the input motion. The computed motions for the EQL, FDEQL, and TFDEQL models and observed motions at $16 \mathrm{~m}$ are shown in Fig. 4. Results show that the peak ground acceleration PGA values resulting from TFDEQL is closest to the observed PGA, and it is only about $5 \%$ lower than that of the observed PGA. However, the PGA value obtained by EQL methods is about $40 \%$ lower than that of the observed PGA. The PGA value obtained by FDEQL methods is about $15 \%$ lower than of the observed PGA. Figure 4 (right) shows that the responses resulting from the FDEQL and TFDEQL models and the observed responses are richer in high-frequency content than those obtained by the EQL model. Therefore, it can be concluded that the TFDEQL model is more efficient than the FDEQL and EQL models in simulating one-dimensional nonlinear site response.

\section{Influence of elastic-shear-strain-threshold- and frequency-dependent soil behavior on site response}

A series of analyses were performed to evaluate the effect of the elastic-strain-threshold- and frequency-dependent soil behavior on propagated ground motion. The soil profile used in the analyses is shown in Fig. 5. The profile was measured at Hainan Island, which is located off the southern coast of China. The shear wave velocity of the bedrock is $570 \mathrm{~m} / \mathrm{s}$. The profile consists of $60 \mathrm{~m}$ of soil sediments with alternating layers of sands and clays, mostly clays. The soil density ranges between 1910 and $2120 \mathrm{~kg} / \mathrm{m}^{3}$. The dynamic properties and the PI were obtained from laboratory tests. To obtain the shear modulus reduction and damping ratio curves, $\mathrm{RC}$ tests were performed on five representative soil specimens. The specimens are numbered sequentially from top to bottom. The $G / G_{\max }-\gamma$ and $D-\gamma$ curves for the five different depth soil specimens are shown in Fig. 6. The profile is subjected to the 1995 Kobe (Japan) earthquake (http://peer.berkeley. edu/peer_ground_motion_database), which is scaled to a maximum acceleration of $0.5 \mathrm{~g}$ prescribed at a hypothetical outcropping of rock. The input motion is shown in Fig. 7.

The EQL, FDEQL, and TFDEQL models are used, respectively, in the analyses to investigate their influence on propagated ground motion. For the EQL model, the ratio of the effective shear strain to the maximum shear strain used is 0.65 .

The computed ground motions at free surfaces, usually of most concern to structural engineers, are shown in Fig. 8. Results show that the values resulting from the TFDEQL and FDEQL methods are about $50 \%$ higher than that of the EQL method. Compared to the FDEQL method, the PGA resulting from the TFDEQL method is about $7 \%$ higher. Frequency content is another important characteristic of ground motions. Figure 8 shows that the responses resulting from FDEQL and TFDEQL soil behavior are richer in high-frequency content, and the Fourier amplitudes for the TFDEQL model are higher than those for the FDEQL model when the frequency is above $3 \mathrm{~Hz}$.

The transfer function from rock to surface, shown in Fig. 9 (left), demonstrates that high-frequency contents amplify greatly for the FDEQL model and especially for 

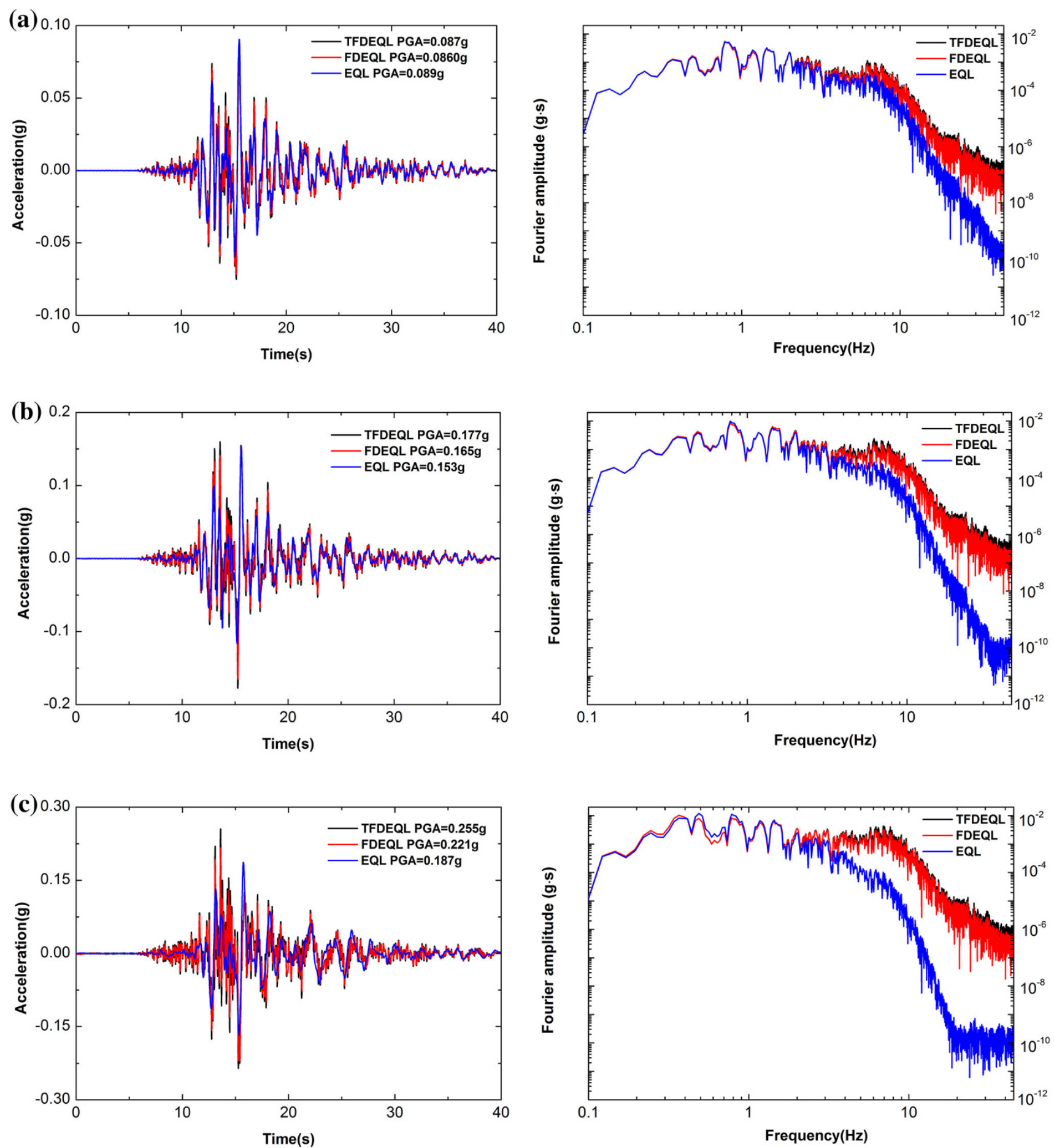

Fig. 10 Time history (left) and Fourier spectra (right) of responses at the free surface for different PGA of input motions: a PGA $=0.05 \mathrm{~g}$; b $\mathrm{PGA}=0.10 \mathrm{~g} ; \mathbf{c} \mathrm{PGA}=0.20 \mathrm{~g} ; \mathbf{d}$ PGA $=0.40 \mathrm{~g}$; and $\mathbf{e} \mathrm{PGA}=0.60 \mathrm{~g}$

the TFDEQL model due to the regulation of high-frequency contents whose strain amplitudes are below the elastic shear strain threshold. Figure 9 (right), on the other hand, depicts the computed $5 \%$-damped acceleration response spectra at the surface. As can be seen, compared to the EQL model, the FDEQL and TFDEQL models generate much higher response at short periods ranging from $0.01 \mathrm{~s}$ to $0.8 \mathrm{~s}$. The maximum values of response spectra and corresponding periods for the three models are listed in Table 1. Table 1 shows that, for the FDEQL and TFDEQL models, the maximum response occurs when the period is $0.25 \mathrm{~s}$; for the $\mathrm{EQL}$ model, the maximum 

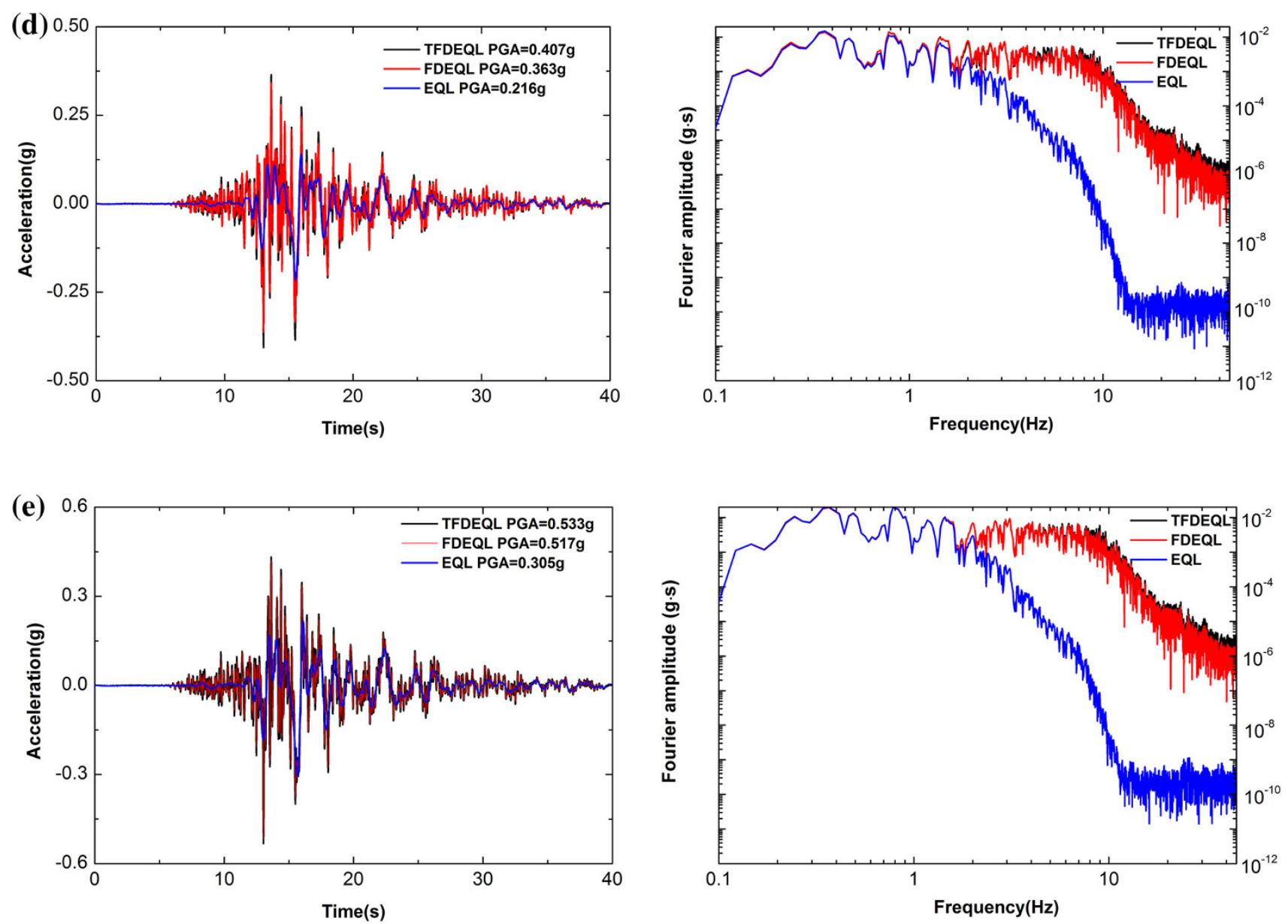

Fig. 10 continued

response occurs when the period is $0.5 \mathrm{~s}$. As a result, the ground motions resulting from the FDEQL and TFDEQL models are harmful to short and stiff structures with short natural periods and especially to nuclear reactor buildings whose frequencies are supposed to range between 4 and $10 \mathrm{~Hz}$ or higher (Kennedy et al. 1980); moreover, the maximum value of response spectra obtained by the TFDEQL model is about $70 \%$ higher than that obtained by the EQL model, and the maximum value obtained by the TFDEQL model is about $35 \%$ higher than that of the FDEQL model.

\section{Soil behavior for the EQL, FDEQL, and TFDEQL models from weak to strong motions}

To investigate the soil behavior for the EQL, FDEQL, and TFDEQL models under different intensities of input ground motions, the PGA of the input ground motions, shown in Fig. 7, was scaled to be $0.05,0.10,0.20,0.40$, and $0.60 \mathrm{~g}$, and a series of soil response analyses were implemented. The soil responses at the free surface and Fourier spectra are shown in Fig. 10. Also, the amplitude transfer functions from rock to surface and $5 \%$-damped surface acceleration response spectra are shown in Fig. 11.

Figure 10 (left) shows that the three models result in almost the same PGA of the computed surface ground motion when the input motion is weak (PGA $=0.05 \mathrm{~g}$ ); however, the differences become apparent as the input motion moves from weak to strong. Also, Fig. 10 (right) indicates that the computed surface ground motions obtained by FDEQL and TFDEQL are much richer in highfrequency contents, and the ground motions obtained by TFDEQL are the richest out of the three models. The transfer functions from rock to surface, shown in Fig. 11 (left), indicate that amplification of high-frequency contents for the EQL model becomes lower and lower with increasing intensities of input motion. In contrast, for the FDEQL and TFDEQL models, amplification of high-frequency contents is always high, and the TFDEQL model always results in the highest amplification factor. Figure 11 (right) shows that the FDEQL and TFDEQL models yield a much higher response than the EQL model in short periods ranging from 0.01 to $0.8 \mathrm{~s}$, and the TFDEQL model yields 

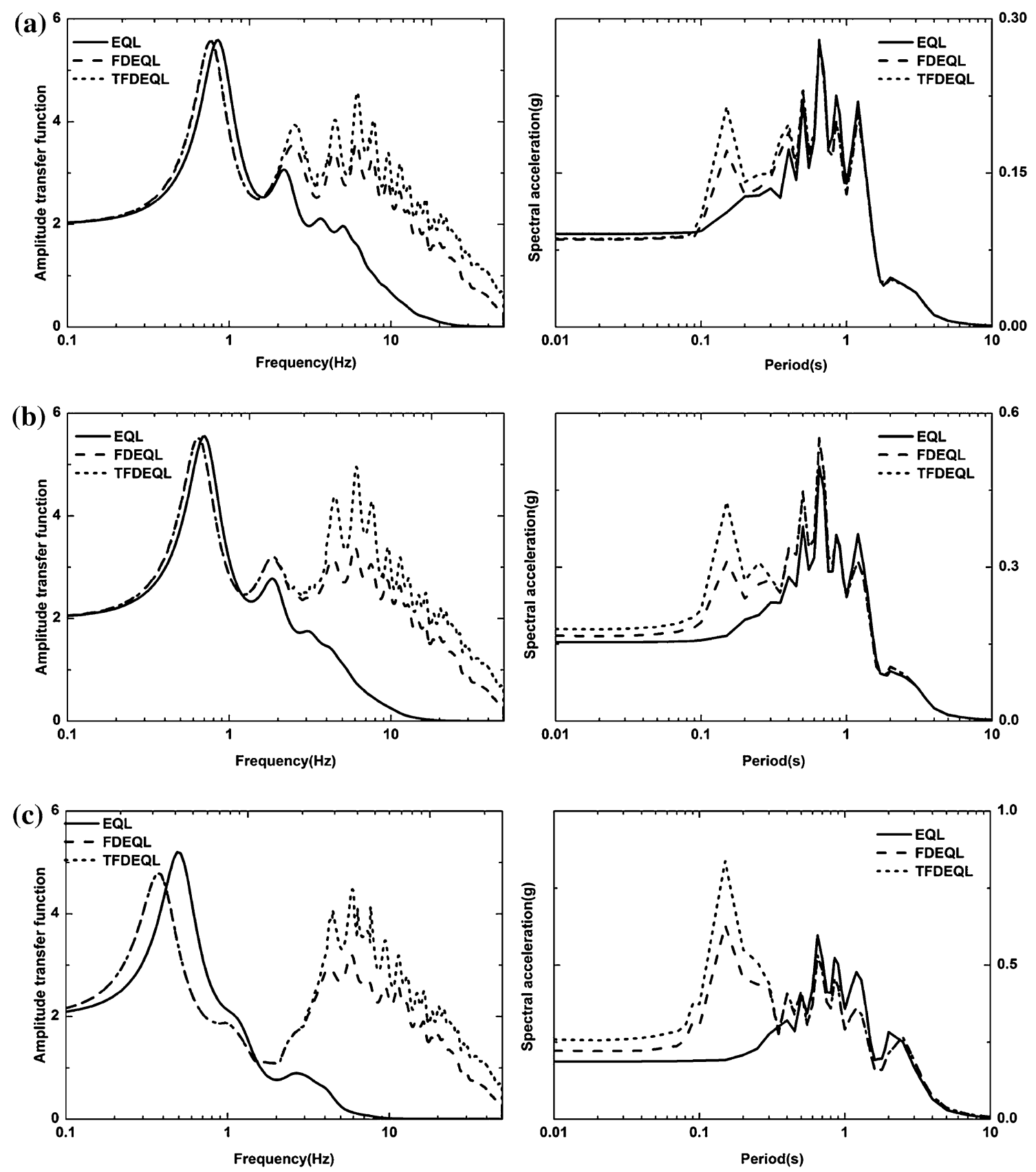

Fig. 11 Amplitude transfer function from rock to surface (left) and $5 \%$ damped surface acceleration response spectra (right) for different values of PGA for input motions: a PGA $=0.05 \mathrm{~g}$; b PGA $=0.10 \mathrm{~g}$; $\mathbf{c}$ PGA $=0.20 \mathrm{~g} ; \mathbf{d}$ PGA $=0.40 \mathrm{~g}$; and e PGA $=0.60 \mathrm{~g}$

a higher response than FDEQL at short periods ranging from 0.01 to $0.3 \mathrm{~s}$, which also proves that the response obtained by the FDEQL and TFDEQL models are richer in high-frequency content, with TFDEQL being the richest. As for the values of the response spectra, the greatest difference is that the TFDEQL model yields $33 \%$ higher values than the FDEQL model at a period of $0.15 \mathrm{~s}$.

Figure 12 depicts the variation of PGA of computed surface ground motion versus PGA of input motion for three models. As can be seen clearly, PGA resulting from the TFDEQL model is highest, and PGA resulting from the
EQL model is lowest as the PGA of input motion is above $0.05 \mathrm{~g}$; the effects of the TFDEQL and FDEQL models on PGA becomes increasingly pronounced as the input motion becomes stronger.

\section{Discussion and conclusion}

To investigate the effects of elastic-shear-strain- and frequency-dependent soil behavior on propagated ground motions, a series of analyses were performed using EQL, 

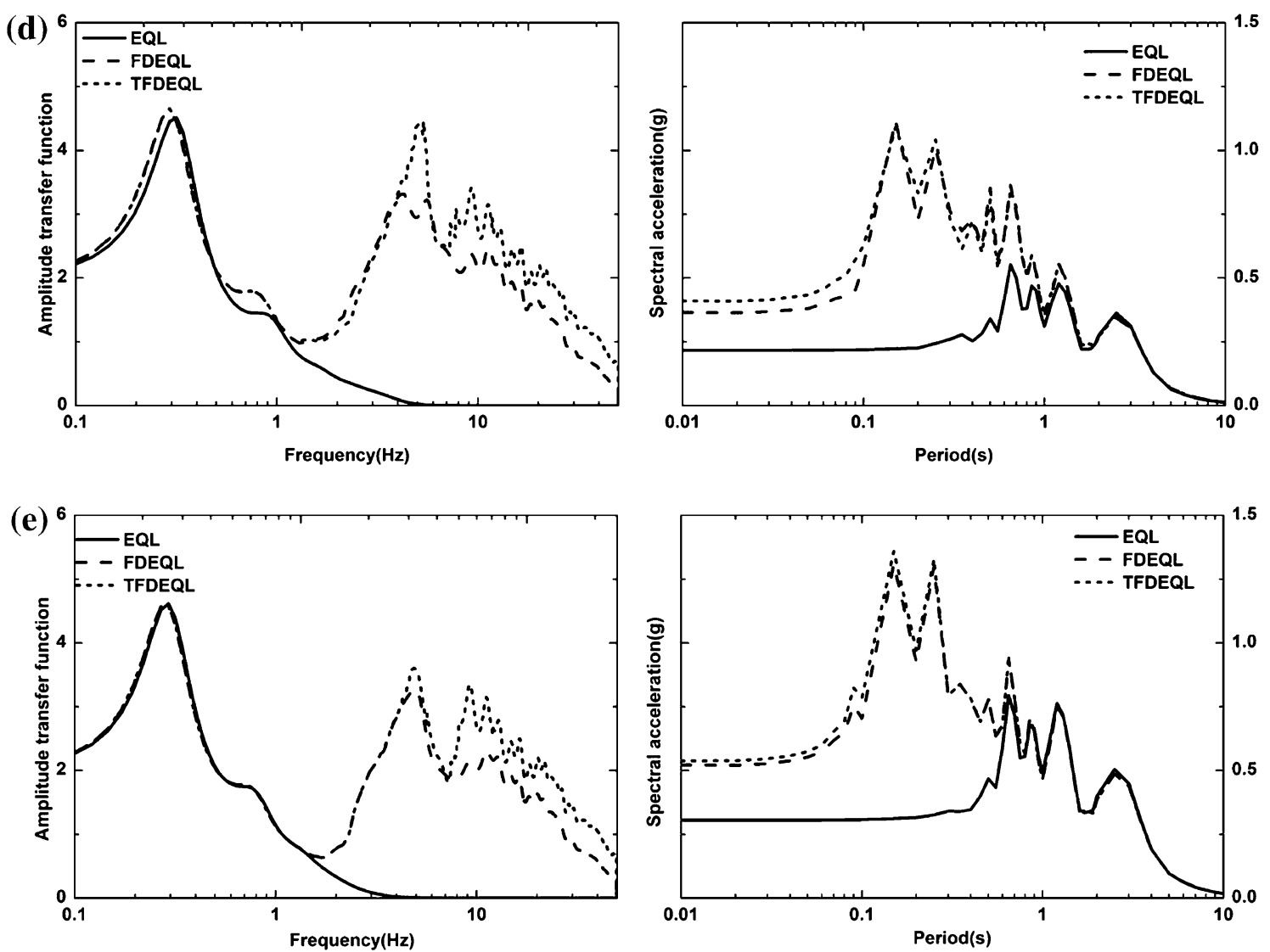

Fig. 11 continued

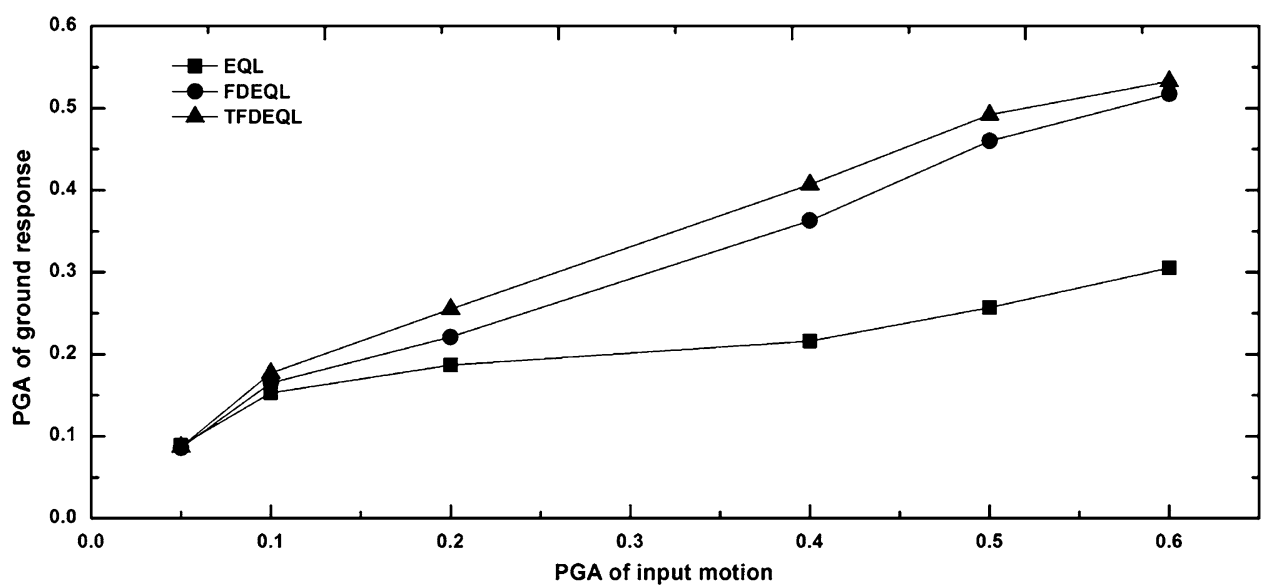

Fig. 12 Comparison of PGA of surface ground response to PGA of input motion for the three models

FDEQL, and TFDEQL models. Figure 10 (a-left) shows that values of PGA obtained by three models are almost the same when the input motion is weak $(\mathrm{PGA}=0.05 \mathrm{~g})$; however, despite the minor differences in PGA, Figs. 10 (a-left) and 11 (a-left) show that the response obtained by the FDEQL and TFDEQL models are richer in high-frequency contents, which are supposed to cause a higher response in structures with short periods, such as nuclear reactor buildings. As the input motion becomes stronger, the difference in PGA for the three models becomes larger. As shown in Fig. 12, the PGA obtained by the TFDEQL model can be $90 \%$ higher than that of the EQL model; the PGA obtained by the FDEQL model can be $80 \%$ higher than that of the EQL model; and the PGA obtained by the TFDEQL model can be $15 \%$ higher than that of the FDEQL model. For the frequency contents characteristic of 
computed ground motion, the same conclusion can be derived as that derived by weak input motion; the response resulting from the FDEQL and TFDEQL models are always richer in high-frequency contents, and the response resulting from the TFDEQL model is the richest. As a result, it can be inferred that the response obtained by the TFDEQL model is always most harmful to short-period structures as proven by Fig. 11 (right). The response spectra obtained by the TFDEQL model can be $33 \%$ higher than that of FDEQL model for some short periods, which may have a great influence on the structure design of short-period structures.

Although the EQL method was first proposed to simulate wave propagation in soil, it has some disadvantages (Sugito 1995; Assimaki and Kausel 2002; Kausel and Assimaki 2002; Yoshida et al. 2002). The FDEQL method was later proposed in order to account for the variation of shear modulus and damping with frequency. Based on the FDEQL soil behavior and strain threshold theory, an improved iterative method, the TFDEQL method, is proposed to account for the high-frequency contents with strain amplitudes below the elastic strain threshold.

A series of analyses involving soft-layered profiles subjected to weak and strong earthquake motions were conducted. Results show that the responses obtained by the FDEQL and TFDEQL models are always richer in highfrequency contents whether or not the input motion is weak, and the response obtained by the TFDEQL model is always richest. The difference in PGA values for the three methods depends on the intensity of input motion; if the input motion is weak, the difference can be neglected, but if the input motion is strong, the difference is pronounced. The ground response obtained by the TFDEQL model is up to $30 \%$ higher than that of the FDEQL model at short periods, which could play an important role in the design of structures with short periods.

Acknowledgments This study is supported by the Science for Earthquake Resilience of China Earthquake Administration (Grant No. XH14060) and the National Natural Science Foundation of China (Grant No. 51478135).

\section{References}

Anderson DG, Richart FE (1976) Effects of straining on shear modulus of calys. J Geotech Geoenviron 102(9):975-987

Assimaki D, Kausel E (2002) An equivalent linear algorithm with frequency and pressure-dependent moduli and damping for the seismic analysis of deep sites. Soil Dyn Earthq Eng 22(9-12): 959-965

Díaz-Rodríguez J, López-Molina J (2008) Strain thresholds in soil dynamics. In: The Proceedings of the 14th World Conference on Earthquake Engineering, Beijing
Guo XX (2012) Dynamic shear modulus and damping ratio for threshold strain in cohesionless soils. Appl Mech Mater 105: 1603-1606

Hashash YMA, Phillips C, Groholski DR (2010) Recent advances in non-linear site response analysis. In: Fifth International Conference in Recent Advances in Geotechnical Eartqhuake Engineering and Soil Dynamics, San Diego

Hsu CC, Vucetic M (2003) Volumetric threshold shear strain for cyclic settlement. J Geotech Geoenviron 130(1):58-70

Idriss IM, Joseph IS (1992) User's manual for SHAKE91: a Computer program for conducting equivalent linear. seismic response analyses of horizontally layered soil deposits. University of California, Davis

Jiang T, Xing H (2007) An equivalent linear method considering frequency-dependent soil properties for seismic response analysis. Chin J Geotech Eng 29(2):218 (in Chinese with English abstract)

Kausel E, Assimaki D (2002) Seismic simulation of inelastic soils via frequency-dependent moduli and damping. J Eng Mech-Asce 128(1):34-47

Kennedy RP, Cornell C, Campbell R, Kaplan S, Perla H (1980) Probabilistic seismic safety study of an existing nuclear power plant. Nucl Eng Des 59(2):315-338

Kim DS, Stokoe KH, Hudson WR (1991) Deformational characteristics of soils at small to intermediate strains from cyclic tests. Research report 1177-3, Center for Transportation Research, Bureau of Engineering Research, University of Texas at Austin

Kwak DY, Jeong CG, Park D, Park S (2008) Comparison of frequency dependent equivalent linear analysis methods. In: The 14th World Conference on Earthquake Engineering, Beijing

Lanzo G, Vucetic M (1999) Effect of soil plasticity on damping ratio at small cyclic strains. Soils Found 39(4):131-141

Lanzo G, Vucetic M, Doroudian M (1997) Reduction of shear modulus at small strains in simple shear. J Geotech Geoenviron 123(11):1035-1042

Miura K, Kobayashi S, Yoshida N (2000) Equivalent linear analysis considering large strains and frequency dependent characteristics. In: 12th world conference on earthquake engineering, Auckland, pp 1832-1837

Ohsaki Y (1994) New introduction of sprctrum analysis of ground motion. Kajima Shuppan, Tokyo

Okur D, Ansal A (2007) Stiffness degradation of natural fine grained soils during cyclic loading. Soil Dyn Earthq Eng 27(9):843-854

Rahhal ME, Lefebvre G (2006) Characterizing Shear Moduli Reduction in Soils Cyclic Behavior. In: Geotechnical Engineering in the Information Technology Age, GeoCongress, pp 1-5

Schnabel PB, Lysmer J, Seed HB (1972) Shake:a computer program for earthquake response analysis of horizontally layered sites. University of California, Berkeley

Sugito M (1995) Frequency-dependent equivalent strain for earthquake response analysis of soft ground. In: The first international conference on earthquake geotechnical engineering, Tokyo, pp 655-660

Vucetic M (1994) Cyclic threshold shear strains in soils. J Geotech Eng 120(12):2208-2228

Vucetic M, Dobry R (1991) Effect of soil plasticity on cyclic response. J Geotech Eng 117(1):89-107

Yoshida N, Kobayashi S, Suetomi I, Miura K (2002) Equivalent linear method considering frequency dependent characteristics of stiffness and damping. Soil Dyn Earthq Eng 22(3):205-222

You HB, Zhao FX, Rong MS (2009) Nonlinear seismic response of horizontal layered site due to inclined wave. Chin J Geotech Eng 31(2):234-240 (in Chinese with English abstract) 